\title{
Programa de estimulação no ambiente educacional para aprimoramento de habilidades subjacentes à leitura
}

\section{Stimulation program in an educational setting for improvement of skills underlying reading}

\author{
Esmeralda Sandra Santos Damasceno ${ }^{1}$ (D), Marisa Sacaloski ${ }^{1}$ (D), Mariana de Oliveira Costa ${ }^{1}$ (D), \\ Adriana de Souza Batista Kida ${ }^{1}$ (1), Clara Regina Brandão de Avila ${ }^{1}$ (1)
}

\section{RESUMO}

Objetivo: elaborar e investigar o efeito de um programa de estimulação de habilidades cognitivo-linguísticas preditoras da decodificação leitora, orientado para a melhora das habilidades subjacentes à leitura. Métodos: pesquisa prospectiva, de análise quantitativa. Participaram 124 crianças de $1^{\circ}$ a $3^{\circ}$ ano do Ensino Fundamental I de uma escola pública de São Paulo, distribuídas em: Grupo Pesquisa - 62 escolares que passaram por intervenção; Grupo Controle: 62 escolares que não receberam estimulação. Avaliaramse individualmente (pré e pós-intervenção) parâmetros de fluência leitora (taxa e acurácia) em tarefa com palavras isoladas. O programa contou com tarefas destinadas a estimular a discriminação auditiva, conhecimento morfossintático, acesso fonológico ao léxico mental, consciência fonológica, conhecimento do código escrito, fluência leitora e o vocabulário visual de palavras e foi construído para aprimorar, em dez sessões de estimulação coletiva, a decodificação e reconhecimento automático de palavras. Resultados: o Grupo Pesquisa apresentou maiores diferenças de acurácia e escore na leitura de palavras, quando comparados os resultados das avaliações pré e pós-intervenção com os do Grupo Controle. Conclusão: o programa promoveu a fluência leitora de escolares do $1^{\circ}$ e $2^{\circ}$ anos do Ensino Fundamental I. Incrementos significativos foram observados para a discriminação auditiva, consciência fonológica e automaticidade leitora, com variações em função do ano escolar.

Palavras-chave: Leitura; Aprendizagem; Educação baseada em competências; Fonoaudiologia; Alfabetização

\begin{abstract}
Purpose: To develop and investigate the effect of a program that stimulates cognitive-linguistic skills predictive of phonological decoding skills aimed at improving of skills underlying reading. Methods: The research design was a prospective cohort study with quantitative analysis. A total of 124 $1 \mathrm{st}$ - 3rd grade children from a public Ensino Fundamental I school in São Paulo, participated in the study, divided into: Research Group - 62 students who underwent intervention; Control Group: 62 students who did not receive stimulation. Reading fluency parameters (rate and accuracy) were individually evaluated (pre- and post-intervention) in a task with isolated words. The program had tasks designed to stimulate auditory discrimination, morphosyntactic knowledge, phonological access to the mental lexicon, phonological awareness, knowledge of the written code, reading fluency and the visual vocabulary of words. It was built to improve decoding and automatic word recognition skills in ten sessions of collective stimulation. Results: The Research Group showed greater differences in accuracy and score in word reading, when comparing the results of pre- and post-intervention assessments, than the Control Group. Conclusion: The program promoted the reading fluency of $1 \mathrm{st}$ and 2 nd grade students. Significant increments were observed for auditory discrimination, phonological awareness and reading automaticity with variations depending on the grade.
\end{abstract}

Keywords: Reading; Learning; Competency-based education; Speech therapy; Literacy

\footnotetext{
Trabalho realizado na Universidade Federal de São Paulo - UNIFESP - São Paulo (SP), Brasil.

${ }^{1}$ Departamento de Fonoaudiologia, Universidade Federal de São Paulo - UNIFESP - São Paulo (SP), Brasil.

Conflito de interesses: Não.

Contribuição dos autores: ESSD elaboração do projeto de pesquisa (mestrado), estudo bibliográfico e metodológico, coleta e análise dos dados, elaboração e revisão do texto final; MS elaboração e revisão do texto final; MOC participação na elaboração do projeto, análise estatística dos dados, participação na construção da discussão dos dados de pesquisa e participação na elaboração do artigo; ASBK coorientadora do projeto de pesquisa que deu origem a esse artigo, estudo metodológico, análise dos dados, elaboração e revisão do texto final; CRBA orientadora do projeto de pesquisa que deu origem a esse artigo, estudo metodológico, análise dos dados, elaboração e revisão do texto final.
}

Financiamento: Conselho Nacional de Desenvolvimento Científico e Tecnológico (CNPq) - Projeto Universal 459953/2014-1 e Coordenação de Aperfeiçoamento de Pessoal de Nível Superior (CAPES) - bolsa de mestrado.

Autor correspondente: Esmeralda Sandra Santos Damasceno. E-mail: hesksandra@gmail.com

Recebido: Julho 28, 2021; Aceito: Novembro 01, 2021 


\section{INTRODUÇÃO}

Os resultados do Sistema de Avaliação da Educação Básica $^{(1)}$ revelaram que $39 \%$ dos alunos do $5^{\circ}$ ano do Ensino Fundamental de escolas públicas brasileiras apresentaram níveis de proficiência de Língua Portuguesa abaixo do esperado, com índices insatisfatórios de alfabetização desde os anos iniciais de escolarização. Essa condição coloca os escolares em risco para falharem em algum momento do percurso escolar. Doze estados brasileiros possuem maior concentração de estudantes do $3^{\circ}$ ano do Ensino Fundamental I com nível 2 ou 3 da escala de proficiência em Língua Portuguesa e, portanto, abaixo do esperado. Além disso, $42 \%$ dos alunos do $9^{\circ}$ ano de Ensino Fundamental de escolas públicas brasileiras apresentaram precariedade no domínio de habilidades básicas relacionadas à proficiência em Língua Portuguesa ${ }^{(1)}$, também estando em risco para desenvolverem níveis mais avançados de proficiência em leitura.

De fato, o Programme for International Student Assessment $t^{(2)}$ mostrou que, aproximadamente $50,0 \%$ dos estudantes brasileiros alcançaram notas abaixo do nível 2 , identificado pela Organização das Nações Unidas (ONU) como "nível mínimo de proficiência" que todas as crianças devem alcançar até o final do Ensino Médio(2).

Este cenário atual da educação brasileira justifica a necessidade de criação de novas estratégias de estimulação, disponíveis para docentes, e que possam ser facilmente aplicadas em sala de aula. Essa é uma das características do Modelo de Resposta à Intervenção (Response to Intervention - RTI).

Esse modelo, assim como outras abordagens, está direcionado para identificar e monitorar escolares em situação de risco para transtornos do aprendizado ${ }^{(3)}$. É uma proposta de atuação conduzida pelo professor no ambiente escolar, que deve estimular, em primeira e universal camada de intervenção, o desenvolvimento de habilidades cognitivas e linguísticas de todos os estudantes, por meio de atividades que favoreçam a prevenção e a identificação mais acurada de riscos para transtornos do aprendizado escolar ${ }^{(3)}$. Essa identificação e o monitoramento tornam possível a superação das dificuldades a tempo e as manifestações de transtornos podem ser mitigadas graças à aplicação de condutas de apoio(4-6).

A possibilidade de adotar um programa baseado nesse modelo de intervenção mostra-se relevante, especialmente por ser proposto para aplicação coletiva no ambiente escolar - sendo assim de mais baixo custo - e, também, por ocorrer em período favorável do desenvolvimento, já que o período de maior plasticidade cerebral, observada no período inicial da escolarização, pode ser aproveitado pela aplicação de estratégias que enfatizam habilidades relacionadas ao processamento fonológico (reconhecidas como melhores preditores do desempenho em decodificação e reconhecimento de palavras escritas) ${ }^{(4)}$. Trata-se, portanto, de uma intervenção de qualidade, composta por procedimentos e estratégias fundamentados em pesquisas científicas, destinados a desenvolver habilidades e competências relacionadas às demandas acadêmicas e, por fim, acelerar a velocidade do aprendizado escolar ${ }^{(3,6)}$.

Tal intervenção pode promover o desenvolvimento de competências leitoras, possibilitando àqueles que apresentam atrasos uma oportunidade de superar suas dificuldades ${ }^{(3,6)}$. A melhora significativa da leitura de palavras reais de baixa frequência e de escrita regular, produtos da aplicação de um programa de remediação fonológica, confirma a eficácia desse tipo de ação em ambiente educacional ${ }^{(3,6,7)}$.

Outros estudos baseados no treinamento de consciência fonológica e fluência também demonstraram efeitos significativos sobre a decodificação leitora ${ }^{(6,8)}$. Progressos no desempenho, em especial nas habilidades fonológicas, mostraram-se capazes de promover a leitura, tanto pela melhora dos índices de exatidão, quanto pelo aumento da velocidade da leitura oral.

O modelo de intervenção educacional universal promove a aquisição das habilidades no tempo oportuno e reduz o número de alterações no aprendizado e consequentes encaminhamentos e intervenções nas áreas correlatas da saúde ${ }^{(3)}$.

A identificação precoce também é crucial para a escola brasileira, pois o índice de prejuízos escolares no Brasil é alto, se comparado à prevalência de transtornos no exterior, onde os sistemas educacionais estão mais estruturados para oferecer o máximo de estimulação a seus alunos. Muitos desses fracassos não decorrem de problemas neurobiológicos, mas de fatores externos, especialmente de restrições no ambiente escolar ${ }^{(2)}$.

Essas evidências motivaram o presente estudo, que teve como objetivo elaborar e estudar o efeito de um programa de estimulação em habilidades cognitivo-linguísticas preditoras da decodificação leitora, com o intuito de melhorar as habilidades subjacentes à leitura e a leitura, propriamente dita.

\section{MÉTODO}

Este estudo quantitativo e prospectivo (follow-up) foi aprovado pelo Comitê de Ética em Pesquisa - CEP/Unifesp (CAAE: 47579715.9.0000.5505, Parecer 1.309.571).

\section{Amostra}

Foram recrutadas 143 crianças (74 meninas e 69 meninos), entre 6 anos e 9 anos e 6 meses, regularmente matriculadas nas três primeiras séries do Ensino Fundamental I de uma escola da rede pública do município de São Paulo (Tabela 1). Para inclusão desses escolares na amostra, adotaram-se os seguintes critérios: assinatura do Termo de Consentimento Livre e Esclarecido (TCLE - pais e/ou responsáveis) e do Termo de Assentimento Livre e Esclarecido (TALE- crianças); frequência mínima de $80 \%$ das sessões de estimulação (mínimo de oito sessões); presença nos momentos da execução das sessões de rastreio em situação pré e pós-intervenção. A perda amostral durante a execução dos procedimentos de pesquisa foi de 19 escolares.

As condições experimentais adotadas para o estudo foram: Grupo Pesquisa (GP), composto por 62 escolares que passaram pela intervenção; Grupo Controle (GC), com 62 escolares que receberam apenas a estimulação escolar durante o período de aplicação da intervenção ao GP(Tabela 1). A adoção desse desenho experimental buscou controlar o sabido efeito da escolarização sobre as variáveis de decodificação leitora, possibilitando compreender melhor o efeito do programa proposto.

Como desejado para o desenho experimental, os grupos não diferiram quando mensuradas as variáveis de controle na situação pré-intervenção (precisão de leitura: $\mathrm{F}=0.106, \mathrm{p}=0.901$, tempo de leitura: $\mathrm{F}=0.213, \mathrm{p}=0.813$ ). Essa igualdade é uma condição fundamental por garantir que eventuais diferenças encontradas sejam efeito direto do programa aplicado. 
Tabela 1. Distribuição percentual dos sujeitos segundo o grupo e a escolaridade $(n=124)$

\begin{tabular}{|c|c|c|c|c|c|c|}
\hline \multirow{2}{*}{ Escolaridade } & \multicolumn{2}{|c|}{ Grupo Controle } & \multicolumn{2}{|c|}{ Grupo Pesquisa } & \multicolumn{2}{|c|}{ Total } \\
\hline & No. & $\%$ & No. & $\%$ & No. & $\%$ \\
\hline $1^{\circ}$. Ano & 23 & $18,6 \%$ & 22 & $17,7 \%$ & 45 & $36,3 \%$ \\
\hline $2^{\circ}$. Ano & 19 & $15,3 \%$ & 20 & $16,1 \%$ & 39 & $31,5 \%$ \\
\hline $3^{\circ}$. Ano & 20 & $16,1 \%$ & 20 & $16,1 \%$ & 40 & $32,2 \%$ \\
\hline Total & 62 & $50,0 \%$ & 62 & $50,0 \%$ & 124 & $100,0 \%$ \\
\hline
\end{tabular}

Legenda: No. = número de participantes; \% = percentual

O estudo foi realizado em três etapas: 1) rastreio pré-intervenção (pré-int); 2) aplicação do procedimento de estimulação das habilidades e competências subjacentes ao aprendizado da decodificação leitora; 3) rastreio pós-intervenção (pós-int). Os rastreios foram realizados com a finalidade de investigar o desempenho dos escolares nas tarefas de processamento fonológico e de leitura. Permitiram monitorar o aproveitamento da estimulação ofertada por meio do programa, na comparação dos momentos pré e pós intervenção $0^{(9)}$. Consistiram nas seguintes tarefas:

- Prova de leitura oral de lista de palavras isoladas: psicometricamente validada, composta por 24 palavras (extensão: $58.3 \%$ de 4-6 letras; 41.7\% 7-8 letras; correspondência letra-som: $52,1 \%$ regulares e $47,9 \%$ irregulares $)^{(10)}$. Os escolares foram instruídos a ler oralmente, de modo rápido e preciso, a lista de palavras isoladas, apresentadas em fonte arial, tamanho 12. Calcularam-se a taxa e acurácia de leitura ${ }^{(11)}$. Foi realizado o cômputo dos acertos obtidos na leitura de 24 palavras $^{(10)}$.

- Teste de discriminação auditiva por figuras: foram apresentados 40 pares de palavras que diferiam entre si por apenas um fonema (pares mínimos), representados em forma de figuras ${ }^{(12)}$. Para cada par mínimo, havia três fichas contendo dois desenhos em cada uma. A criança avaliada ouvia a pronúncia de duas palavras do par, que poderiam ser iguais ou diferentes e, em seguida, indicava a ficha que continha figuras correspondentes às palavras ouvidas. Desempenhos acima de $80 \%$ foram considerados apropriados.

- Tarefa de nomeação rápida de objetos: prova psicometricamente validada, estimula o acesso fonológico ao léxico mental ${ }^{(13)}$, composta por um cartão contendo seis figuras (ovo, pão, bola, sol, chave, garfo) que se repetem em ordem aleatória, seis vezes no cartão, totalizando 36 aparições por cartão. As crianças foram instruídas a nomear o mais rapidamente possível cada uma das figuras. Cada acerto correspondeu a 1 ponto e o tempo total de execução foi registrado em segundos. Omissões e repetições foram consideradas erros.

- Tarefa de exclusão fonêmica-CONFIAS (Consciência Fonológica Instrumento de Avaliação Sequencial): Subteste psicometricamente estudado ${ }^{(14)}$, composto por dois itens de treino e seis itens de teste. As tarefas de consciência fonológica foram escolhidas, pois são excelentes preditores e contribuem para a exatidão e fluência leitora ${ }^{(8)}$. O avaliador solicitou ao escolar que suprimisse um fonema específico de uma dada palavra. Foi atribuído 1 ponto para cada acerto e calculado o escore a partir da soma desses acertos.
O rastreio foi aplicado individualmente, em sala apropriada, disponibilizada pela escola. Os testes foram precedidos de uma etapa de treino. Os participantes do GP e do GC passaram simultaneamente pelo rastreio nos momentos pré e pós-intervenção para que se controlasse o possível efeito da progressão escolar. Destaca-se que os escolares do GC participaram do Programa de Estimulação posteriormente à coleta dos dados, na etapa pós-intervenção do estudo, respeitando os procedimentos éticos.

\section{Programa de Estimulação}

Composto por atividades que envolveram as habilidades de discriminação auditiva de palavras; consciência fonológica; acesso fonológico ao léxico mental; estimulação da leitura de palavras e de texto. Foi aplicado por uma fonoaudióloga duas vezes por semana, em sala de aula, perfazendo dez sessões de 50 minutos. A opção pela instrução sistemática e intensiva (frequência e duração) procurou atender às recomendações de evidências científicas ${ }^{(6,7,9)}$

O programa foi estruturado da seguinte forma: as cinco primeiras sessões estimularam a discriminação auditiva, consciência fonológica, conhecimento do código gráfico (reconhecimento de letras), leitura oral conjunta, vocabulário visual, acesso fonológico ao léxico; as sessões de seis a dez estimularam a consciência fonológica, conhecimento do código escrito (valor sonoro de letras), leitura oral conjunta, vocabulário visual e acesso fonológico ao léxico. Essas tarefas encontram-se brevemente descritas a seguir:

- Discriminação auditiva de palavras: desenvolvida para aprimorar a identificação de contrastes fonêmicos no contexto de palavras. Apresentaram-se sequências de quatro palavras, compostas por uma palavra-alvo e outras duas palavras similares distintas por apenas um fonema, caracterizando, portanto, uma oponência mínima ou máxima $^{(12,15,16)}$. As oponências foram selecionadas para que as crianças identificassem contrastes fonêmicos a partir das pistas sonoras de frequência e duração.

- Consciência fonológica: as tarefas detiveram-se na estimulação da consciência metafonológica em seus níveis silábico e fonêmico. Privilegiaram-se atividades fonêmicas, bem como tarefas baseadas nas habilidades de identificação, supressão ou síntese ${ }^{(17)}$. Cada item de estimulação previsto ao longo das sessões contou com a escolha de uma palavra-alvo (resposta correta) e duas opções competitivas (alta competição, caracterizada por oponência mínima ou máxima com o fonema ou sílabaalvo, estímulo de baixa competição sem similaridade fonológica).

- Acesso fonológico ao léxico mental: tarefas idealizadas para estimular a velocidade e a precisão no acesso fonológico 
ao léxico mental ${ }^{(18-20)}$. As pranchas de nomeação rápida das sessões iniciais foram compostas por dois pares de estímulos que representavam palavras caracterizadas por oponências máximas ou mínimas e um dos estímulos era fonologicamente diferente dos demais. As pranchas aplicadas nas sessões finais favoreceram a evocação da nomeação a partir de uma pista fonológica previamente fornecida (Ex: Todas as figuras da folha começam com /f/).

- Reconhecimento de letras: consistiu na nomeação de letras, atribuição de seus valores sonoros e reconhecimento de letras a partir de seu valor sonoro ${ }^{(20-23)}$.

- Leitura de palavras e textos: foi realizada a leitura oral conjunta de histórias dos livros da Coleção Estrelinha da Editora Ática, conhecida pelo controle da complexidade ortográfica (O pato e o sapo, O galo maluco, O peru de peruca, $\mathrm{A}$ onça e a anta, $\mathrm{O}$ caracol viajante, $\mathrm{A}$ arara cantora, $\mathrm{O}$ mistério da lua, $\mathrm{O}$ barulho fantasma, $\mathrm{O}$ susto do periquito, Um palhaço diferente). Os textos ofertados foram organizados de modo a aumentar progressivamente o grau de dificuldade ao longo das sessões de ortografia transparente à opaca. Para a criação de um vocabulário visual de palavras, selecionaram-se 96 palavras dos textos apresentados (9,6 palavras por texto), que eram apresentadas após a leitura do livro. Eram palavras de alta frequência, que variaram quanto à extensão (di, tri e polissilábicas) e regularidade ortográfica, a fim de garantir a frequência de exposição desejada para o reconhecimento automático da forma visual das palavras. A aplicação das palavras foi cumulativa ao longo das sessões, permitindo que a frequência de exposição garantisse, assim, o efeito desejado. A partir da aplicação das tarefas propostas, foi possível estimar o risco para alterações no desempenho. Para os escolares do primeiro ano, em que não há parâmetros de análise ofertados pela Prova de Leitura de Itens, considerou-se risco a ausência de qualquer possibilidade de leitura de palavras por rota fonológica, independentemente da fluência observada. Já para escolares do segundo e terceiro ano, foi adotado como critério a classificação do desempenho como inferior ou médio inferior.

Todas as atividades propostas foram projetadas em sala com o uso de datashow, contendo pranchas com figuras representativas dos estímulos. Os alunos receberam instruções orais e cada tarefa foi precedida por uma etapa de treino. Folhas de resposta foram distribuídas como forma de engajar os escolares na realização das tarefas.

\section{Tratamento estatístico}

Para análise estatística, realizou-se estudo da normalidade dos dados - aplicação do teste Kolmogorov-Smirnov (K-S); estudo de comparação entre as variáveis de fluência leitora no rastreio pré-intervenção (pré-int) e no rastreio pós-intervenção (pós-int) para cada um dos grupos - Teste de Wilcoxon; estudo de comparação da evolução dos grupos - Teste de Mann-Whitney. Utilizou-se o programa SPSS (Statistical Package for Social Sciences) em sua versão 22.0. Consideraram-se estatisticamente significantes os resultados com valor de $\mathrm{p}<0,05$.

\section{RESULTADOS}

Após a aplicação do programa de estimulação, verificou-se que ambos os grupos apresentaram resultados superiores de taxa e acurácia no rastreio pós-intervenção (pós-int). Entretanto, apenas o Grupo Pesquisa revelou melhora na tarefa de leitura das 24 palavras psicometricamente selecionadas (Tabela 2).

O Grupo Pesquisa (GP) apresentou maior diferença entre os rastreios pré-intervenção e pós-intervenção para as variáveis relacionadas à precisão de leitura. As maiores diferenças, sempre positivas, indicaram que, após a intervenção com o programa de resposta à intervenção, o GP apresentou aumento significativamente maior dos índices de acurácia e dos acertos na leitura das 24 palavras isoladas, em comparação ao Grupo Controle (GC). A análise comparativa do desempenho nos rastreios pré-intervenção e pós-intervenção para os grupos estudados foi realizada de modo a investigar resultados decorrentes da estimulação das habilidades e capacidades subjacentes à decodificação e reconhecimento de palavras (Tabela 3).

O GP apresentou melhor desempenho no rastreio pósintervenção em todos os aspectos investigados, enquanto o GC não progrediu significativamente, nesse período, nas habilidades de discriminação auditiva e consciência fonológica.

O GP mostrou aumento significativamente maior dos acertos como efeito da aplicação do programa sobre as variáveis de discriminação auditiva e de consciência fonológica no $1^{\circ}$ ano. Por sua vez, no $2^{\circ}$ ano, observou-se o aumento significativo de acertos somente para o GP, quando considerada a variável consciência fonológica.

Para o $3^{\circ}$ ano, não houve diferenças significativas entre o desempenho do GP e GC, quando comparadas as diferenças de desempenho nos rastreios pré-intervenção e pós-intervenção de avaliação (Tabela 4).

A análise comparativa dos resultados das variáveis de decodificação e fluência leitora, em função da escolaridade, obtidos pelos escolares do GP e GC, teve como objetivo analisar os efeitos do programa de estimulação nas diferentes séries escolares do estudo. Ao observar a análise do $1^{\circ}$ e $2^{\circ}$ anos, verificou-se que o GP apresentou maior diferença de desempenho entre o rastreio pós-intervenção e pré-intervenção que o GC, para as variáveis taxa, acurácia e para os acertos na leitura das 24 palavras psicometricamente selecionadas. Não houve efeito significativo do treinamento sobre as variáveis de decodificação leitora para os escolares do $3^{\circ}$ ano (Tabela 5).

Quanto à identificação de risco para os transtornos da leitura obtidos nos rastreios pré-intervenção e pós-intervenção para o Grupo Pesquisa (Quadro 1), verificou-se que o percentual de risco para os transtornos da leitura, verificado a partir do desempenho dos escolares na Prova de Leitura de Itens Isolados, indicou diminuição desse percentual, quando comparados os rastreios pré-intervenção e pós-intervenção para todos os anos escolares.

\section{DISCUSSÃO}

O Programa de Resposta à Intervenção (RTI) tem sido utilizado para o estímulo ao aprendizado, favorecendo a prevenção e a identificação precoce e acurada de risco de transtornos do aprendizado escolar. Para tanto, as tarefas aplicadas estimulam as habilidades do processamento fonológico e do conhecimento do código escrito como forma de promover a fluência leitora 
Tabela 2. Resultados comparativos das variáveis de decodificação e fluência leitora no rastreio pré-intervenção e rastreio pós-intervenção) pelo grupo de pesquisa e grupo controle

\begin{tabular}{|c|c|c|c|c|c|c|}
\hline & & & & & & \\
\hline & Variáveis & (Pré-int) & (Pós-int) & & & Resultado \\
\hline & Taya & $216(210)$ & & & * & \\
\hline & Acurácia & $13,3(18,4)$ & $19,3(22,6)$ & $Z=-2,294$ & $0,000^{*}$ & $\mathrm{M} 2>\mathrm{M} 1$ \\
\hline & Acertos_24p & $6(7)$ & $8(9)$ & $Z=-2,113$ & $0,000^{*}$ & $\mathrm{M} 2>\mathrm{M} 1$ \\
\hline GC & Taxa & $21,1(18,6)$ & $24,9(22,5)$ & $Z=-3,663$ & $0,000^{*}$ & $\mathrm{M} 2>\mathrm{M} 1$ \\
\hline & Acurácia & $11,6(16,2)$ & $15,1(20,1)$ & $Z=-3,784$ & $0,000^{*}$ & $\mathrm{M} 2>\mathrm{M} 1$ \\
\hline & Acertos_24p & $6(7)$ & $6(7)$ & $Z=-0,750$ & 0,161 & $\mathrm{M} 1=\mathrm{M} 2$ \\
\hline
\end{tabular}

Teste: Wilcoxon, nível de significância estatística: $p=0,05\left(^{*}\right)$

Legenda: GP = grupo pesquisa; GC = grupo controle; dp = desvio padrão; Taxa = total de palavras lidas por minuto; Acurácia = total de palavras lidas corretamente por minuto; Acertos_24p = total de acertos na leitura das 24 palavras psicometricamente selecionadas; pré-int = pré-intervenção; pós-int = pós-intervenção; Z = resultado do teste de Wilcoxon; $\mathrm{M}=$ momento

Tabela 3. Resultados comparativos das variáveis de habilidades e capacidades subjacentes à decodificação e fluência leitora nos rastreios préintervenção e pós-intervenção obtidas pelo grupo de pesquisa e grupo controle

\begin{tabular}{|c|c|c|c|c|c|}
\hline & \multirow{3}{*}{ Variáveis } & \multicolumn{2}{|c|}{ Rastreio } & \multirow{3}{*}{ Teste Wilcoxon } & \multirow{3}{*}{ Resultado } \\
\hline & & (pré-int) & (pós-int) & & \\
\hline & & Média (dp) & Média (dp) & & \\
\hline \multirow[t]{4}{*}{ GP } & Disc_Aud & $27,3(15,8)$ & $28,5(15,9)$ & $0,004^{*}$ & pós-int > pré-int \\
\hline & NR_temp & $43,6(14,4)$ & $38,1(12,6)$ & $0,000^{*}$ & pós-int < pré-int \\
\hline & NR_erros & $7,3(3,6)$ & $6(2,72)$ & $0,001^{*}$ & pós-int < pré-int \\
\hline & CF & $3,1(1,9)$ & $4,4(1,91)$ & $0,000^{*}$ & pós-int > pré-int \\
\hline \multirow[t]{4}{*}{ GC } & Disc_Aud & $37,6(2,7)$ & $37,8(2,3)$ & 0,742 & pré-int $=$ pós-int \\
\hline & NR_temp & $42,9(12,9)$ & $40,3(13,7)$ & $0,005^{*}$ & pós-int $<$ pré-int \\
\hline & NR_erros & $7,6(2,6)$ & $6,68(2,6)$ & $0,004^{*}$ & pós-int $<$ pré-int \\
\hline & CF & $3,56(1,9)$ & $3,82(1,79)$ & 0,059 & pré-int = pós-int \\
\hline
\end{tabular}

Teste de Wilcoxon, nível de significância estatística: $p=0,05\left(^{*}\right)$

Legenda: GP = grupo de pesquisa; GC = grupo controle; Disc_Aud: total de acertos na prova de discriminação auditiva; NR_tempo = tempo dispendido em tarefa de nomeação rápida em segundos; NR_erros = total de erros cometidos em tarefa de NR; CF = desempenho em consciência fonológica (tarefa de exclusão fonêmica); pré-int = pré-intervenção; pós-int = pós-intervenção

Tabela 4. Resultados comparativos entre grupo de pesquisa e grupo controle quanto à diferença de desempenho entre os rastreios pós-intervenção e pré-intervenção em tarefas de investigação das habilidades e capacidades subjacentes a decodificação e fluência leitora por ano escolar

\begin{tabular}{|c|c|c|c|c|c|}
\hline \multirow{3}{*}{ Ano Escolar } & \multirow{3}{*}{ Variáveis } & \multicolumn{2}{|c|}{ Grupo } & \multirow{3}{*}{ Teste Mann-Whitney } & \multirow{3}{*}{ Resultadc } \\
\hline & & GP & GC & & \\
\hline & & $\begin{array}{l}\text { Dif (pré-int)-(pós-int) } \\
\text { (dp) }\end{array}$ & $\begin{array}{l}\text { Dif (pré-int)-(pós-int) } \\
\text { (dp) }\end{array}$ & & \\
\hline \multirow[t]{4}{*}{$1^{\circ}$. } & Disc_Aud & $2,04(1,5)$ & $0,13(1,5)$ & $0,000^{*}$ & $\mathrm{GP}>\mathrm{GC}$ \\
\hline & NR_tempo & $-4,50(7,7)$ & $5,54(11,5)$ & 0,953 & $\mathrm{GP}=\mathrm{GC}$ \\
\hline & NR_erros & $-0,91(3,5)$ & $0,59(3,4)$ & 0,846 & $\mathrm{GP}=\mathrm{GC}$ \\
\hline & CF & $1,08(1,2)$ & $0,61(1,3)$ & $0,015^{\star}$ & $\mathrm{GP}>\mathrm{GC}$ \\
\hline \multirow[t]{4}{*}{$2^{\circ}$. } & Disc_Aud & $0,22(1,4)$ & $0,45(2,2)$ & 0,806 & $\mathrm{GP}=\mathrm{GC}$ \\
\hline & NR_tempo & $-6,72(16,5)$ & $3,05(10,9)$ & 0,087 & $\mathrm{GP}=\mathrm{GC}$ \\
\hline & NR_erros & $-1,11(3,2)$ & $-0,05(3,0)$ & 0,239 & $\mathrm{GP}=\mathrm{GC}$ \\
\hline & CF & $1,72(1,3)$ & $0,40(1,1)$ & $0,004^{*}$ & $\mathrm{GP}>\mathrm{GC}$ \\
\hline \multirow[t]{4}{*}{$3^{\circ}$. } & Disc_Aud & $0,05(1,3)$ & $-0,09(1,5)$ & 0,688 & $\mathrm{GP}=\mathrm{GC}$ \\
\hline & NR_tempo & $-5,42(11,8)$ & $-4,66(6,1)$ & 0,469 & $\mathrm{GP}=\mathrm{GC}$ \\
\hline & NR_erros & $-1,94(2,5)$ & $-1,85(1,9)$ & 0,789 & $\mathrm{GP}=\mathrm{GC}$ \\
\hline & CF & $0,89(1,9)$ & $-0,23(0,8)$ & 0,065 & $\mathrm{GP}=\mathrm{GC}$ \\
\hline
\end{tabular}

Teste Mann-Whitney, nível de significância estatística: $\mathrm{p}=0,05\left(^{*}\right)$

Legenda: GP = grupo de pesquisa; GC = grupo controle; Disc_Aud = total de acertos na prova de discriminação auditiva; NR_tempo = tempo dispendido em tarefa de nomeação rápida em segundos; NR erros = total de erros cometidos em tarefa de NR; CF = desempenho em consciência fonológica (tarefa de exclusão fonêmica); Dif (pré-int)-(pós-int): diferença de desempenho entre o rastreio pré-intervenção e o rastreio pós-intervenção; dp = desvio padrão

nos anos escolares iniciais ${ }^{(24)}$. Assim, a estimulação constante e adequada das habilidades subjacentes à decodificação deve promover a automaticidade da leitura ${ }^{(23,24)}$.

No presente estudo, após o treinamento, verificou-se aumento da velocidade e acurácia para os escolares do GP e para os escolares do GC, mostrando que a escolaridade influencia a automaticidade leitora (Tabela 2). Quando comparados os desempenhos de taxa e acurácia entre os momentos pré-intervenção e pós-intervenção, foi possível constatar que o GP apresentou maior índice de acertos para as 24 palavras psicometricamente 
Tabela 5. Resultados comparativos entre grupo de pesquisa e grupo controle quanto à diferença de desempenho entre os rastreios pré-intervenção e pós-intervenção em tarefas de decodificação e fluência leitora por ano escolar.

\begin{tabular}{|c|c|c|c|c|c|}
\hline \multirow{3}{*}{ Ano Escolar } & \multirow{3}{*}{ Variáveis } & \multicolumn{2}{|c|}{ Grupo } & \multirow{3}{*}{ Teste Mann-Whitney } & \multirow{3}{*}{ Resultadc } \\
\hline & & GP & GC & & \\
\hline & & $\begin{array}{l}\text { Dif (pré-int)-(pós-int) } \\
\text { (dp) }\end{array}$ & $\begin{array}{l}\text { Dif (pré-int)-(pós-int) } \\
\text { (dp) }\end{array}$ & & \\
\hline \multirow[t]{3}{*}{$1^{\circ}$} & Taxa & $5,91(7,8)$ & $1,57(3,5)$ & $0,020^{*}$ & $\mathrm{GP}>\mathrm{GC}$ \\
\hline & Acurácia & $1,96(4,2)$ & $0,72(2,3)$ & $0,023^{*}$ & $\mathrm{GP}>\mathrm{GC}$ \\
\hline & Acertos_24p & $1,65(2,7)$ & $0,08(0,7)$ & $0,004^{*}$ & $\mathrm{GP}>\mathrm{GC}$ \\
\hline \multirow[t]{3}{*}{$2^{\circ}$} & Taxa & $7,24(6,1)$ & $3,20(4,4)$ & $0,028^{*}$ & $\mathrm{GP}>\mathrm{GC}$ \\
\hline & Acurácia & $8,65(7,3)$ & $1,94(5,3)$ & $0,002^{*}$ & $\mathrm{GP}>\mathrm{GC}$ \\
\hline & Acertos_24p & $4,11(3,7)$ & $0,10(3,5)$ & $0,002^{*}$ & $\mathrm{GP}>\mathrm{GC}$ \\
\hline \multirow[t]{3}{*}{$3^{\circ}$. } & Taxa & $5,29(8,4)$ & $7,07(6,7)$ & 0,215 & $\mathrm{GP}=\mathrm{GC}$ \\
\hline & Acurácia & $8,09(7,1)$ & $8,05(8,0)$ & 1 & $\mathrm{GP}=\mathrm{GC}$ \\
\hline & Acertos_24p & $2,47(2,8)$ & $1,71(4,0)$ & 0,282 & $\mathrm{GP}=\mathrm{GC}$ \\
\hline
\end{tabular}

Teste: Mann-Whitney, nível de significância estatística: $p=0,05\left(^{*}\right)$

Legenda: GP = grupo de pesquisa; GC = grupo controle; Taxa = total de palavras lidas por minuto; Acurácia = total de palavras lidas corretamente por minuto; Acertos_24p = total de acertos na leitura das 24 palavras psicometricamente selecionadas; Dif (pré-int)-(pós-int) = diferença de desempenho entre o rastreio pré-intervenção e o rastreio pós-intervenção; $d p=$ desvio padrão

Quadro 1. Total de escolares do grupo de pesquisa identificados com potencial risco para transtornos da leitura segundo rastreio por meio da Prova de Leitura de Itens Isolados, em função do ano escolar

\begin{tabular}{|c|c|c|c|c|c|}
\hline \multirow{2}{*}{$\begin{array}{c}\text { Ano } \\
\text { Escolar }\end{array}$} & \multirow{2}{*}{$\begin{array}{c}\text { Níveis de } \\
\text { Desempenho** }\end{array}$} & \multicolumn{2}{|c|}{ Momento do Rastreio } & \multicolumn{2}{|c|}{ Percentual de Risco } \\
\hline & & (pré-int) & (pós-int) & (pré-int) & (pós-int) \\
\hline \multirow[t]{2}{*}{$1^{\circ}$ ano } & Não lê & 17 & 10 & $73 \%$ & $43 \%$ \\
\hline & Lê & 6 & 13 & & \\
\hline \multirow[t]{5}{*}{$2^{\circ}$ ano } & Inferior & 9 & 5 & $68 \%$ & $47 \%$ \\
\hline & Médio Inferior & 4 & 4 & & \\
\hline & Médio & 3 & 1 & & \\
\hline & Médio superior & 3 & 7 & & \\
\hline & Superior & 0 & 2 & & \\
\hline \multirow[t]{5}{*}{$3^{0}$ ano } & Inferior & 7 & 6 & $45 \%$ & $35 \%$ \\
\hline & Médio Inferior & 2 & 1 & & \\
\hline & Médio & 2 & 5 & & \\
\hline & Médio superior & 6 & 1 & & \\
\hline & Superior & 3 & 7 & & \\
\hline \multirow[t]{2}{*}{ TOTAL } & Com risco & 39 & 26 & $63 \%$ & $41 \%$ \\
\hline & Sem risco & 23 & 36 & $37 \%$ & $58 \%$ \\
\hline
\end{tabular}

** Dados em negrito indicam os escolares considerados de risco para os transtornos da leitura. Para os escolares do primeiro ano, em que não há parâmetros de análise ofertados pela Prova de Leitura de Itens Isolados, considerou-se risco a ausência de qualquer possibilidade de leitura de palavras por rota fonológica, independentemente da fluência observada. Já para escolares do segundo e terceiro, ano foi adotado como critério a classificação do desempenho como inferior ou médio inferior.

Legenda: pré-(int) = pré-intervenção; pós-(int) = pós-intervenção

selecionadas após as atividades, demonstrando o efeito do treinamento das habilidades pelo programa, pois as palavras selecionadas são de baixa frequência, sendo necessário que o leitor principiante faça uso da rota fonológica para realizar a leitura da palavra. Esses resultados sugerem a efetividade do Programa de Resposta à Intervenção sobre a decodificação e fluência leitora. Não foram encontrados na literatura estudos ou programas de estimulação que, semelhantes a este, estimulassem todas as habilidades, competências e conhecimentos selecionados no presente trabalho, bem como se destinassem exclusivamente aos escolares das três séries iniciais do Ensino Fundamental. No entanto, a literatura evidencia a eficácia de ações de intervenção destinadas a estimular habilidades do processamento fonológico ou da linguagem com repercussões positivas sobre a decodificação leitora $^{(8,20,21)}$. Os programas estruturados a partir de abordagens multidimensionais de estimulação apresentam resultados mais efetivos para a promoção da decodificação leitora do que aqueles que utilizam apenas o treinamento de habilidades ou competências subjacentes isoladas ${ }^{(3,8,23,25)}$. Os efeitos do treinamento de habilidades, competências e conhecimentos linguísticos e do código escrito relacionados à decodificação, bem como o treinamento explícito da leitura, também se mostraram eficientes na promoção da capacidade de decodificação e favorecimento da automaticidade no reconhecimento de palavras. Outros autores utilizaram um programa de resposta à intervenção com escolares com dificuldade de leitura e escrita do $3^{\circ}$ e $4^{\circ}$ anos do Ensino Fundamental, para verificar a eficácia do programa RTI, e estimularam as habilidades de consciência fonológica, vocabulário, fluência e compreensão. Os alunos apresentaram melhora nas tarefas de leitura e escrita, revelando que o programa RTI é eficaz e eficiente para os escolares em diferentes aspectos e favorece o desenvolvimento da leitura e escrita, a exemplo do observado na presente pesquisa ${ }^{(3)}$.

Outro programa de intervenção utilizou a nomeação automática rápida e a leitura ${ }^{(19)} \mathrm{e}$ demonstrou melhora na identificação de sílabas e de fonema final e medial, adição e combinação de 
sílabas, repetição de não palavras, leitura de palavras reais e pseudopalavras.

Outras pesquisas mostraram que o treinamento conjunto de habilidades subjacentes, relacionadas à consciência fonológica e ao conhecimento das letras do alfabeto e seus valores sonoros, é efetivo para promoção do aprendizado da leitura e escrita, principalmente nos primeiros anos escolares ${ }^{(8,23)}$.

As habilidades de consciência fonológica e o vocabulário exercem maior influência que o nível de inteligência em crianças nos primeiros anos do aprendizado da leitura e escrita ${ }^{(8)}$.

Não foram encontrados, na literatura, estudos cujos treinamentos das habilidades subjacentes à decodificação não tenham promovido a capacidade de ler palavras de modo preciso e automático ${ }^{(26)}$. Assim, os resultados obtidos na presente pesquisa confirmam os achados da literatura, o que permitiu concluir que programas de estimulação baseados no aprimoramento e treinamento de habilidades, competências e/ou conhecimentos subjacentes à decodificação leitora podem ser benéficos, em especial, para a precisão da leitura. Além disso, a literatura atesta que seus benefícios são potencializados quando se associa o treinamento de diversas habilidades e/ou conhecimentos implicados na decodificação ${ }^{(7,8,23,24,26)}$.

A análise dos resultados dos efeitos do treinamento desenvolvido sobre os componentes do processamento da informação fonológica e da linguagem (Tabela 3) revelou que o GP apresentou melhora da discriminação auditiva, da precisão e velocidade de acesso lexical e da consciência fonológica. A comparação do perfil de resposta dos grupos, no entanto, demonstra que a escolarização também promoveu o desempenho do $\mathrm{GC}$, exceto para a discriminação auditiva e consciência fonológica. Comparativamente, a consciência fonológica promoveu ganhos significativamente maiores para o GP. O treinamento da discriminação auditiva, proposto a partir do paradigma baseado em estratégias para desenvolvimento da discriminação contrastiva dos fonemas (oponências máximas e mínimas) no contexto da palavra, mostrou-se capaz de aprimorar essas habilidades auditivas e de percepção da fala após as dez sessões de estimulação para o GP. Tal estimulação é eficaz para promover o aprendizado das propriedades do sistema fonológico ${ }^{(16)}$, base para o processamento da informação fonológica requerida pela decodificação leitora ${ }^{(7)}$. $\mathrm{O}$ treinamento da discriminação fonêmica para escolares em início de instrução leitora, ou mesmo com dificuldades no aprendizado do código alfabético, promove as habilidades auditivas e de decodificação leitora ${ }^{(26)}$.

As dificuldades na percepção do menor segmento da fala pode comprometer o aprendizado da leitura, pois a associação grafema-fonema leva a criança a fazer leitura de qualquer palavra $^{(24)}$. Os resultados e a literatura consultada permitiram supor que a instrução combinada de habilidades auditivas com as de consciência fonológica, comprovadamente eficazes no presente estudo, proporcionou aos escolares do GP aprimorar capacidades para identificar as unidades de fala (sílabas e fonemas) requeridas para a compreensão e aprendizado do código gráfico e essenciais para o desenvolvimento da rota fonológica da leitura.

A análise dos resultados referentes à eficácia do treinamento sobre o desempenho da decodificação e fluência leitora nos diferentes anos escolares investigados mostrou que os participantes do GP matriculados no $1^{\circ}$ e $2^{\circ}$. anos se beneficiaram da intervenção, apresentando aumento expressivamente superior dos valores de taxa, de acurácia e do total de acertos para a leitura das 24 palavras psicometricamente selecionadas, quando comparados ao GC (Tabela 4).

Os primeiros anos destinados ao ensino da leitura $\left(1^{\circ}\right.$ e $2^{\circ}$ anos escolares) requerem do aprendiz o aumento da consciência fonológica e a apropriação progressiva do conhecimento das letras. Esse aprendizado, por sua vez, permite aos escolares identificar as conexões entre a palavra escrita e sua forma fonológica, construindo, progressivamente, hipóteses sobre as regras que regem o código gráfico ${ }^{(8,24)}$. Essas associações permitem o surgimento da leitura por rota fonológica, altamente dependente do processamento das informações fonológicas. Em séries mais avançadas, o escolar passa a utilizar cada vez menos a leitura por essa rota, uma vez que a própria experiência leitora permite a ampliação de um vocabulário visual e o progressivo domínio no reconhecimento de unidades morfêmicas, que conduzem o surgimento da leitura por rota lexical e promovem a automaticidade leitora ${ }^{(27,28)}$. Confirmam essa hipótese os estudos que indicam que os aspectos relacionados ao processamento da informação fonológica, em especial a consciência fonológica, são excelentes preditores e contribuem para a exatidão e fluência leitora. Contudo, seu papel mostrou-se mais relevante em séries iniciais e suas contribuições decresceram à medida que a escolaridade aumentou ${ }^{(27)}$. Por outro lado, a consciência morfológica, que contribui para o progressivo aumento do uso da leitura por rota lexical, passa a ser necessária para a compreensão e identificação dos padrões do sistema ortográfico da língua e é mais requerido a partir do $2^{\circ}$ e $3^{\circ}$ anos do Ensino Fundamental ${ }^{(24)}$.

Os resultados da presente pesquisa confirmaram os da literatura, o que permitiu supor a influência positiva do treinamento multidimensional proposto para a promoção da decodificação e reconhecimento automático de palavras. No entanto, a eficácia mostrou-se restrita aos primeiros anos de escolarização, em que a instrução sobre o código gráfico ocorreu, sugerindo que o programa atuou mais especificamente sobre as habilidades e competências requeridas para o processamento da informação fonológica, que ancorou o desenvolvimento da leitura por rota fonológica, que, por sua vez, ao perder relevância com o passar da escolarização, em razão do uso mais frequente do reconhecimento por rota lexical, justificaria os resultados observados.

Apesar dos resultados promissores, uma limitação do presente trabalho deve ser considerada: escolares com dificuldades na apropriação da leitura podem demorar a ampliar o uso da rota lexical, mesmo diante da escolarização e do aumento da experiência leitora. Evidências obtidas em pesquisas demonstraram que, na ausência da acurácia leitora, há maior restrição para o crescimento do vocabulário visual ${ }^{(24)}$. Nesse sentido, estudos complementares deverão ser realizados exclusivamente com escolares considerados de risco para os transtornos da leitura.

A avaliação do efeito do programa sobre seus desempenhos leitores permitirá verificar se uma possível melhora do processamento da informação fonológica e da consequente acurácia leitora poderia ocorrer e, consequentemente, promover o reconhecimento automático de palavras, em consonância com as postulações dos pesquisadores ${ }^{(8)}$.

Os resultados do treinamento dos componentes do processamento da informação fonológica e da linguagem, em função do ano escolar, demonstraram que os escolares do $1^{\circ}$ ano do GP submetidos ao programa beneficiaram-se significativamente mais que os escolares do GC, para tarefas de discriminação auditiva e consciência fonológica. Por sua vez, 
os escolares do $2^{\circ}$ ano do GP também apresentaram maiores ganhos que o GC para a consciência fonológica. Escolares do $3^{\circ}$. ano apresentaram mesmo ganho de desempenho para tarefas de processamento da informação fonológica e da linguagem, demonstrando, diferentemente do previsto, a ausência de um efeito de superioridade do programa sobre o próprio ensino escolar (Tabela 5). Essa análise associou-se aos achados anteriormente discutidos, que evidenciaram a eficácia de treinamentos envolvendo a discriminação auditiva e consciência fonológica para escolares de séries iniciais do Ensino Fundamental, que passaram a identificar mais acurada e conscientemente as características fundamentais dos fonemas, resultando em melhora na organização do sistema de representação fonológica ${ }^{(5,6,18)}$. No entanto, a própria escolarização também promoveu essas habilidades e competências, que perderam sua relevância com a progressão escolar ${ }^{(20,27)}$.

Muitas das análises sobre os modelos de resposta à intervenção apresentaram seus resultados longitudinais a partir da apresentação de informações que permitiram verificar a redução dos riscos para transtornos do aprendizado como produto da intervenção educacional realizada. É sabido, no entanto, que apenas estudos experimentais do tipo "ensaios clínicos", em que variáveis podem ser controladas, permitiram obter comprovações de que as mudanças observadas fossem decorrentes exclusivamente do programa de estimulação proposto.

Quanto aos resultados quantitativos da redução de risco para transtornos da leitura, observada em função do ano escolar, a análise do total de escolares identificados com potencial risco para os transtornos da leitura demonstrou a redução do percentual de risco para todos os anos escolares investigados, quando analisados os resultados do GP (Quadro 1). Observouse, em um plano geral, que o índice de risco para transtornos da leitura já se mostrou alto no rastreio pré-intervenção, mesmo considerados os critérios de análise obtidos a partir de uma amostra vasta e randomizada, representativa do sistema escolar da cidade de São Paulo ${ }^{(13)}$. Esses índices de desempenho, ainda que excessivamente altos, estão em consonância com os dados observados em provas de avaliação das competências de leitura de escolares brasileiros ${ }^{(2,29)}$.

A comparação dos percentuais de riscos observados nos rastreios pré-intervenção e pós-intervenção permitiu verificar que o programa auxiliou na redução de riscos para transtornos da leitura. Essa redução, no entanto, foi mais eficiente para o $1^{\circ}$ ano do Ensino Fundamental e decresceu, progressivamente, em função do avanço da escolarização. Esse achado está em consonância com a percepção de que o programa é capaz de estimular as habilidades e competências implicadas no processamento fonológico da informação, mais relevante no período inicial da apropriação do código gráfico ${ }^{(8,27)}$. Os altos índices de risco ainda no rastreio pós-intervenção sugerem a necessidade de utilização de programas de intervenção mais específicos, longos e intensivos para redução ainda mais significativa desses percentuais. Programas de Resposta à Intervenção são concebidos como métodos multicamadas, a fim de reduzir, progressivamente, os índices de alteração do aprendizado escolar ${ }^{5,3}$. Ainda que tenha demonstrado sua eficácia, o programa não atingiu os percentuais desejados de redução esperados, segundo proposto pelo Education Department's Institute of Education Sciences ${ }^{(9)}$. É esperada a permanência de aproximadamente $15 \%$ da população na faixa de risco para o aprendizado, após a primeira intervenção coletiva. Cabe ressaltar que os índices propostos aplicam-se ao sistema americano de ensino, no qual as alterações no momento inicial mostraram-se significativamente menores que as encontradas na realidade brasileira, como observado na presente pesquisa.
A continuidade do estudo para a concepção de um programa completo de intervenção educacional, que envolva a atuação universal (camada 1) e a atuação específica com escolares que mantiverem riscos para o aprendizado da leitura (camada 2), faz-se necessária para que se possa atingir com mais eficácia a promoção da leitura e minimização de riscos de alteração na apropriação do código escrito.

Os resultados encontrados se deveram à intensidade da estimulação, instrução docente e ao fato de os grupos terem sido submetidos a essa intervenção no mesmo momento ${ }^{(3,30)}$.

\section{CONCLUSÃO}

O programa de estimulação composto por atividades destinadas a estimular componentes do processamento da informação fonológica e da linguagem foi efetivo, pois se mostrou capaz de promover o desempenho em decodificação leitora. Os componentes do processamento da informação fonológica e da linguagem que responderam positivamente à estimulação proposta pelo programa, independentemente do efeito da instrução escolar, foram: a discriminação auditiva de palavras e a consciência fonológica. Assim, é possível dizer que a promoção dessas habilidades se mostrou eficaz e que suas estimulações no contexto geral do programa foram capazes de auxiliar o processo de aprender a ler.

A consciência fonológica foi a habilidade do processamento fonológico que melhor respondeu, quando observado o desempenho dos escolares que cursavam os anos iniciais de escolarização ( $1^{\circ}$ e $2^{\circ}$ ano do Ensino Fundamental). Os resultados positivos do treinamento da habilidade de discriminação auditiva podem ter ocorrido em razão das atividades elaboradas envolverem contrastes auditivos entre fonemas, baseando-se na identificação de diferenças de frequência e duração por oponências, o que pode ter promovido maior atenção à fonologia da língua.

Por fim, o cuidado metodológico na determinação do GC, cuja participação na intervenção ocorreu somente após o término da estimulação do GP, tornou possível identificar precisamente a eficácia exclusiva do programa de estimulação, neutralizando os efeitos da própria escolarização. É importante destacar que as análises mostraram que a escolaridade, por si só, foi capaz de promover habilidades (acesso fonológico ao léxico), conhecimentos (consciência morfossintática, conhecimento de letras e domínio da relação grafo-fonêmica) e competências leitoras.

\section{AGRADECIMENTOS}

Agradecemos ao Conselho Nacional de Desenvolvimento Científico e Tecnológico (CNPq) - Projeto Universal 459953/2014-1, e à Coordenação de Aperfeiçoamento de Pessoal de Nível Superior (CAPES). Ambas as Fundações apoiaram a execução desta pesquisa.

\section{REFERÊNCIAS}

1. INEP: Instituto Nacional de Estudos e Pesquisas Anisio Teixeira. SAEB: Sistema de Avaliação da Educação Básica 2017 [Internet]. Brasília: INEP/MEC; 2017 [citado em 2020 Maio 20]. Disponível em: http://portal.inep.gov.br/educacao-basica/saeb/resultados 
2. INEP: Instituto Nacional de Estudos e Pesquisas Anisio Teixeira. PISA: Programa Internacional de Avaliação de Estudantes. Relatório Brasil no PISA 2018 [Internet]. Brasília: INEP/MEC; 2019 [citado em 2020 Maio 20]. Disponível em: http://download.inep.gov.br/acoes_internacionais/ pisa/documentos/2019/relatorio_pisa_2018_preliminar.pdf

3. Hoover WA, Tunmer WE. The simple view of reading: three assessments of its adequacy. Remedial Spec Educ. 2018;39(5):304-12. https://doi. org/10.1177/0741932518773154.

4. Steves AE, Walker MA, Vaughn S. The effects of reading fluency interventions on the reading fluency and reading comprehension performance of elementar students with learning disabilities: a synthesis of the research from 2001 to 2014. J Learn Disabil. 2017;50(5):576-90.

5. Hollman AKW, Schmitt BA, Bradfield TA, Rodriguez MC, Mcconnell SR. Redefining individual growth and deselopment indicators phonological awareness. J Learn Disabil. 2015;48(5):495-510. http:// dx.doi.org/10.1177/0022219413510181.

6. Al Otaiba S, Connor CM, Folsom JS, Wanzek J, Greulich L, Schatschneider C, et al. To wait in tier 1 or intervene immediately: a randomized experiment examining first-grade response to intervention in reading. Except Child. 2014;81(1):11-27. http://dx.doi. org/10.1177/0014402914532234.

7. Rakhlin NV, Mourgues C, Cardoso-Martins C, Kornev AN, Grigorenko EL. Orthographic processing is a key predictor of reading fluency in good and poor readers in a transparent orthography. Contemp Educ Psychol. 2019;56:250-61. http://dx.doi.org/10.1016/j.cedpsych.2018.12.002. PMid:31798206.

8. Tilanus EAT, Segers E, Verhoeven L. Predicting responsiveness to a sustained reading and spelling intervention in children with dyslexia. Dyslexia. 2019;25(2):190-206. http://dx.doi.org/10.1002/ dys. 1614.

9. Balu R, Zhu P, Doolittle F, Schiller E, Jenkins J, Gersten R. Evaluation of Response to Intervention Practices for Elementary School Reading. Washington, DC: National Center for Education Evaluation and Regional Assistance, Institute of Education Sciences, U.S. Department of Education; 2015. p. 308-41.

10. Lúcio PS, Moreira HC, Kida ASB, Carvalho CAF, Pinheiro AMV, Mari JJ, et al. Word decoding task: item analysis by IRT and within-group norms. Psic: Teor e Pesq. 2018.34:e3437. https://doi. org/10.1590/0102.3772e3437.

11. Ávila CRB, Kida ASB, Carvalho CAF. Avaliação da comunicação mediada pela escrita. In: Lamônica DAC, Britto DBO, organizadores. Tratado de linguagem: perspectivas contemporâneas. Ribeirão Preto: Book Toy; 2016. p. 91-105.

12. Santos CB, Mota HB, Keske SM, Attoni TM. Habilidades de discriminação auditiva em crianças com desvios fonológicos evolutivos. Pró-Fono (Online). 2010;22(3):311-6. https://doi.org/10.1590/S010456872010000300026 .

13. Lúcio PS, Kida ABS, Carvalho CAF, Cogo-Moreira H, Avila CRB. Prova de nomeação rápida de figuras para crianças: evidências de validade e normas intragrupo. Psico-USF. 2017;22(1):35-47. http:// dx.doi.org/10.1590/1413-82712017220104.

14. Godoy DMA, Moreira HC. Evidências da Estrutura Fatorial e Precisão de Tarefas de Consciência Fonêmica (TCFe). Paidéia. 2015;25(62):36372.

15. Moojen S, Lamprecht RR, Santos RM, Freitas GM, Brodacz R, Siqueira M, et al. CONFIAS - Consciência Fonológica: Instrumento de Avaliação Sequencial. São Paulo: Casa do Psicólogo; 2007.
16. Appezzato MM, Hackerott MMS, Ávila CRB. Tarefa de discriminação de fala com pseudopalavras. CoDAS. 2018;30(2):1-8. http://dx.doi. org/10.1590/2317-1782/20182017030.

17. Albuquerque A, Martins AM. Habilidades iniciais de alfabetização em Português: pesquisa transcultural em Portugal e no Brasil. Psico-USF. 2017;22(3):437-48. http://dx.doi.org/10.1590/141382712017220305 .

18. Elhassan Z, Crewther SG, Bavin EL. The contributionn of phonological awareness to reading fluency and its individual sub-skills in readers aged 9- to 12-years. Front Psychol. 2017;8:533.

19. Santos B. Programa de intervenção com a nomeação automática rápida e leitura [Estudo Piloto]. Marília: Universidade Estadual Paulista; 2017.

20. Verhoeven L, van Leeuwe J, Irausquin R, Segers E. The unique role of lexical accessibility in predicting kindergarten emergent literacy. Read Writ. 2016;29(4):591-608. http://dx.doi.org/10.1007/s11145015-9614-8. PMid:27073294.

21. Vadasy PF, Sanders EA. Incremental learning of difficult words in story contexts: the role of spelling and pronouncing new vocabulary. Read Writ. 2015;28(3):371-94. http://dx.doi.org/10.1007/s11145-0149529-9.

22. Miles KP, Ehri CL. Orthographic mapping facilitates sight word memory and vocabulary learning. In: Kilpatrick DA, Joshi RM, Wagner RK, organizadores. Reading development and difficulties. USA: Springer; 2019.p. 63-82. http://dx.doi.org/10.1007/978-3030-26550-2_4.

23. Ehri LC. Orthographic mapping in the acquisition of sight word reading, spelling memory, and vocabulary learning. Sci Stud Read. 2014;18(1):5-21. http://dx.doi.org/10.1080/10888438.2013.819356.

24. Germano GD, Capellini SA. Avaliação das Habilidades Metafonológicas (PROHFON): caracterização e comparação do desempenho em escolares. Psicol Reflex Crit. 2015;28(2):378-87. http://dx.doi.org/10.1590/16787153.201528218.

25. Stainthorp R. Dyslexia and Remediation/ Education: Where next? In: Wydell T, organizadores. Current developmental disorders reports. USA: Springer; 2014. p. 260-66.

26. Schaars MMH, Segers E, Verhoeven L. Word decoding development during phonics instruction in children at risk for dyslexia. Dyslexia. 2017;23(2):141-60. http://dx.doi.org/10.1002/dys.1556. PMid:28470910.

27. Monteiro SM; Soares M. Processos cognitivos na leitura inicial: relação entre estratégias de reconhecimento de palavras e alfabetização. Educação e Pesquisa. 2014;40(2):449-66. http://dx.doi.org/10.1590/ S1517-97022014005000006.

28. Takashima A, Hulzink I, Wagensveld B, Verhoeven L. Emergence of representations through repeated training on pronouncing novel letter combinations leads to efficient reading. Neuropsychologia. 2016;89:14-30. http://dx.doi.org/10.1016/j.neuropsychologia.2016.05.014. PMid:27192222.

29. Andrade OVCA, Andrade PE, Capellini SA. Como identificar e Intervir com crianças de risco para os transtornos de aprendizagem. São Paulo: Pulso editorial; 2014. Modelo de Resposta à intervenção; p. $185-296$.

30. Leite RCD, Brito LRM, Reis VOM, Pinheiro AMV. Consciência fonológica e fatores associados em crianças no início da alfabetização. Rev Psicopedagogia. 2018;35(108):306-17. 